\title{
Efecto del silicio como posible elemento reactivo en la protección frente a la oxidación a elevada temperatura del acero inoxidable AISI 304
}

\author{
E. Otero $^{(*)}$, F.J. Pérez ${ }^{(*)}$, M.P. Hierro ${ }^{(*)}$, C. Gómez $_{(* *)}^{(*)}$, F. Pedraza ${ }^{(*)}$, J.L. de Segovia ${ }^{(* *)}$ \\ y E. Román ${ }^{(* *)}$
}

Resumen Se estudia la influencia de silicio incorporado a la aleación mediante implantación iónica de $1 \times 10^{15}$ iones $/ \mathrm{cm}^{2}$ a $150 \mathrm{keV}$ en el crecimiento de la capa protectora de oxidación basada en $\mathrm{Cr}_{1,3} \mathrm{Fe}_{0,7} \mathrm{O}_{3}$ y en espinelas enriquecidas en manganeso, $\mathrm{Mn}_{1,5} \mathrm{Cr}_{1,5} \mathrm{O}_{4}$, tras someter a un acero inoxidable austenítico AISI 304 a oxidación a $1.173 \mathrm{~K}$ y presión atmosférica de aire durante $144 \mathrm{~h}$. La presencia de pequeñas cantidades de silicio en las capas más externas del material promueve el transporte de cromo durante los primeros estadios de la oxidación. Además, la implantación iónica parece ejercer un efecto beneficioso contra la descarburación de la aleación.

Palabras clave: Oxidación a alta temperatura. Acero inoxidable. Implantación iónica.

The influence of silicon as a possible reactive element in the protection against high temperature oxidation of AISI 304 stainless steel

Abstract The influence of silicon incorporated into the alloy by means of ion implantation of $1 \times 10^{15}$ ions $/ \mathrm{cm}^{2}$ at $150 \mathrm{keV}$ on the protective scale development based upon $\mathrm{Cr}_{13} \mathrm{Fe}_{07} \mathrm{O}_{3}$ and manganese-enriched spinels, $\mathrm{Mn}_{1.5} \mathrm{Cr}_{1.5} \mathrm{O}_{4}$ after oxidation of an austenitic AISI 304 stainless steel at $1,173 \mathrm{~K}$ and atmospheric pressure of air for $144 \mathrm{~h}$ has been studied. The presence of small quantities of silicon at the outermost layers of the alloy promotes transport of chromium during the early stages of oxidation. Further, ion implantation seems to play a beneficial role against decarburization of the alloy.

Keywords: High temperature oxidation. Stainless steel. Ionic implantion.

\section{INTRODUCCIÓN}

Numerosas aleaciones de base hierro, níquel y/o cobalto basan su protección frente a la corrosión a elevada temperatura en la creación de capas de $\mathrm{Cr}_{2} \mathrm{O}_{3}, \mathrm{Al}_{2} \mathrm{O}_{3}$ o de $\mathrm{SiO}_{2}$. Estos óxidos son termodi-

(*) Dpto. de Ciencia de los Materiales e Ingeniería Metalúrgica. Fac. de Ciencias Químicas. Univ. Complutense de Madrid. 28040-Madrid (España).

(**) Instituto Torres Quevedo. (CSIC). Serrano, 144. 28006Madrid (España).

(***) Instituto de Ciencia de Materiales. (CSIC). Campus UAM. Canto Blanco. Madrid (España). námicamente muy estables respecto al metal, y los procesos de transporte a través de dichas capas son generalmente lentos (1). Sin embargo, para temperaturas superiores a $850-900{ }^{\circ} \mathrm{C}$, el $\mathrm{Cr}_{2} \mathrm{O}_{3}$ puede continuar reaccionando con el oxígeno formando la especie volátil $\mathrm{CrO}_{3}$, lo que limita la aplicación de estas aleaciones en largos períodos de servicio (2). Por tanto, para temperaturas superiores y en medios ricos en oxígeno se emplean aleaciones que desarrollen capas superficiales de $\mathrm{Al}_{2} \mathrm{O}_{3}$ o de $\mathrm{SiO}_{2}$ (3). No obstante, la presencia de una capa continua de $\mathrm{SiO}_{2}$ puede actuar como un plano de debilitamiento, debido a las transformaciones $\alpha-\beta$ de la cristobalita formada a alta temperatura (4). 
Numerosos autores han estudiado el efecto beneficioso de los elementos reactivos mediante la adición de itrio y de elementos de las denominadas tierras raras o de sus óxidos, así como mediante la implantación iónica de los primeros (5 y 6). Aunque no se conocen cuáles son los mecanismos exactos que producen este efecto beneficioso, se cree que el elemento reactivo $a$ ) actúa como un impedimento físico en los límites de grano, $b$ ) ancla el óxido a la aleación, $c$ ) provoca la aniquilación de vacantes, $d$ ) reduce las tensiones de crecimiento de la capa de oxidación, $e$ ) promueve la oxidación selectiva del cromo y $f$ ) induce la formación de una capa de óxido de grano fino (7). Sin embargo, apenas se ha prestado atención a la implantación de iones de silicio, aunque, recientemente, Hou y Stringer proponían que este elemento podría actuar como un elemento reactivo favoreciendo el anclaje de la capa de oxidación a la aleación y promoviendo el transporte de cromo para formar dicha capa (8).

En este trabajo se presentan los resultados de la oxidación a $1.173 \mathrm{~K}$ y presión atmosférica de aire de un acero inoxidable austenítico $18 \mathrm{Cr} 8 \mathrm{Ni}$ tras haberle sido implantada una dosis de $1 \times 10^{15}$ iones $/ \mathrm{cm}^{2}$ de silicio a $150 \mathrm{keV}$.

\section{PARTE EXPERIMENTAL}

Se han utilizado muestras de acero inoxidable AISI 304 facilitado por ACERINOX, S.A. de tamaño $15 \times 3,5 \times 1 \mathrm{~mm}$. Posterior al corte se realizó el acabado superficial hasta SiC\#600. Tras la preparación superficial, se pesaron las probetas en una balanza Metler AE100, con precisión de 0,0001 g y se tomaron las dimensiones de las mismas con un calibre.

Las probetas se implantaron en el CAI de implantación iónica de la UCM, con un voltaje de aceleración de los iones generados en la fuente de $150 \mathrm{keV}$, y al haz acelerado se le hizo incidir sobre el blanco con una desviación de $7^{\circ}$ respecto a la normal, para evitar el efecto channeling. La densidad de corriente alcanzada en la implantación fue de $800 \mathrm{nA} / \mathrm{cm}^{2}$ manteniéndose el flujo hasta alcanzar la dosis de $1 \times 10^{15}$ iones $/ \mathrm{cm}^{2}$.

Para caracterizar la implantación iónica realizada, se efectuaron análisis mediante la espectroscopía de electrones Auger (AES) con un equipo JEOL JAMP-10S Auger Microprobe, trabajando a $3 \mathrm{kV}$ y $50 \mu \mathrm{A}$, a un vacío de hasta $10^{-10}$ torr.

Una vez implantadas las muestras, se sometieron a ensayos de oxidación discontinuos en aire a 1.773 K durante $144 \mathrm{~h}$.

Para caracterizar los productos de corrosión se aplicaron las técnicas de microscopía electrónica de barrido (SEM), microanálisis EDS y difracción de rayos $\mathrm{X}(\mathrm{DRX})$.

\section{RESULTADOS Y DISCUSIÓN}

La figura 1 muestra los espectros AES del material implantado con silicio con dosis de hasta $1 \times$ $10^{15}$ iones $/ \mathrm{cm}^{2}$. Se observa, en primer lugar, una presencia masiva de carbono como principal elemento contaminante de la muestra, identificándose, además, silicio $(92 \mathrm{eV})$ y oxígeno $(510 \mathrm{eV})$. Después de sucesivos bombardeos con argón, se produce la desaparición de los picos de carbono y de oxígeno, con una mejor definición del resto de los picos. A partir de los análisis cuantitativos de los espectros, parece ser que el silicio difunde hasta la parte más externa del material, creándose un valle composicional a cierta distancia de la superficie, hasta alcanzar un 3,5\% en masa, ya en el interior del acero. Un factor importante a tener en cuenta, es que si el pico del silicio debe aparecer a $92 \mathrm{eV}$, su desplazamiento a la izquierda, puede indicar la formación de un $\mathrm{SiO}_{2}$ no estequiométrico $(76 \mathrm{eV})$.

Como ya se indicó anteriormente, los estudios cinéticos de oxidación se realizaron mediante ensayos discontinuos en horno hasta $144 \mathrm{~h}$, lo que se muestra en la figura 2. De dicha figura podemos deducir que la implantación de silicio, en las dosis efectuadas, produce un efecto beneficioso en la resistencia a la oxidación a elevada temperatura, a $900^{\circ} \mathrm{C}$. Inicialmente las curvas muestran un trazado casi-lineal hasta las $8 \mathrm{~h}$ de experimentación, tiempo que podríamos denominar de incubación; lo que da lugar a una capa de productos de oxidación, protectora, disminuyendo el exponente de la ley del orden de 0,5 a 0,25 .

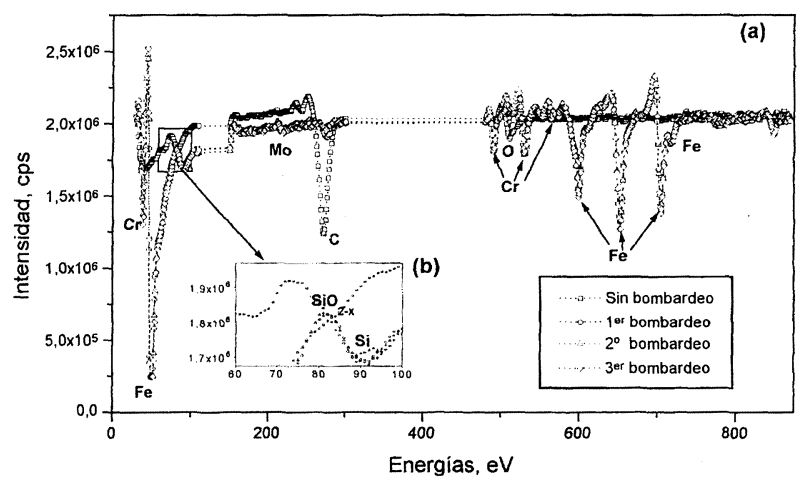

FIG. 1.- a) Espectros Auger del acero AISI 304 implantado con $1 \times 10^{15}$ iones $/ \mathrm{cm}^{2}$ de silicio a 150 $\mathrm{keV}$ sin bombardeo de argón (trazo cuadrados) y con bombardeos sucesivos de argón (círculos, triángulos y rombos, respectivamente). $b$ ) Detalle de la región del pico Auger de silicio.

FIG. 1.- a) Auger spectra for the $1 \times 10^{15} \mathrm{ions} / \mathrm{cm}^{2}$ Si-implanted AISI 304 steel at $150 \mathrm{keV}$ without argon bombardment (squares) and with the consecutive argon bombardments (circles, triangles and diamonds, respectively). b) Silicon Auger peak detail. 


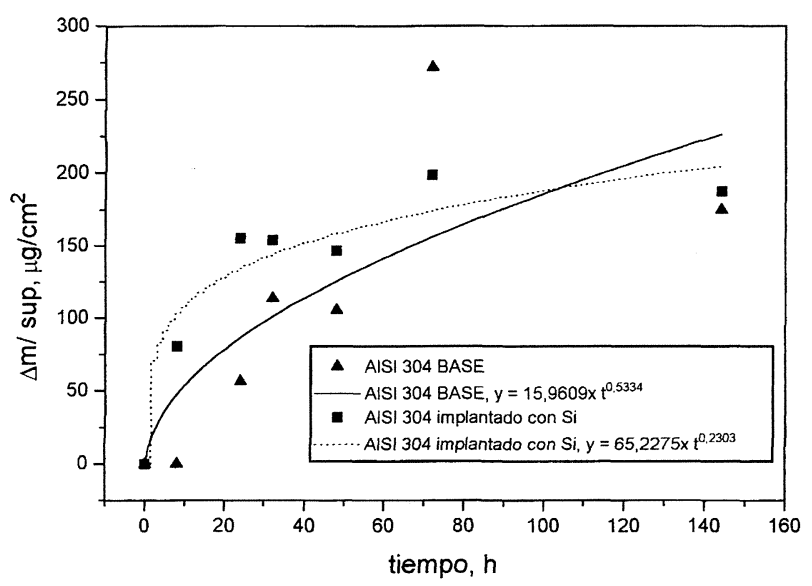

FIG. 2.- Valores experimentales y leyes cinéticas obtenidas para el acero AISI 304 base (trazo continuo) y para el mismo acero implantado con $1 \times 10^{15}$ iones $/ \mathrm{cm}^{2}$ de silicio a $150 \mathrm{keV}$ (trazo discontinuo) sometidos a oxidación a $1.173 \mathrm{~K}$ y presión atmosférica de aire durante $144 \mathrm{~h}$.

FIG. 2.- Experimental values and the kinetics laws for parent AISI 304 steel (solid line) and for the $1 \times 10^{15}$ ions $/ \mathrm{cm}^{2}$ Si-implanted AISI 304 steel (dot line) oxidized at 1,173 $\mathrm{K}$ and atmospheric pressure of air for $144 \mathrm{~h}$.

La figura 3 muestra la morfología superficial del material oxidado hasta $144 \mathrm{~h}$ de ensayo. En él, se observó el desarrollo de la capa de productos oxidados siguiendo las líneas de desbaste superficial, sobre la cual, y de forma local, crecían una serie de rosetas que presentaban un enriquecimiento apreciable en manganeso (Fig. 3b) con respecto al resto de la superficie oxidada (Fig.3c). Para poder analizar las especies que se formaron en la superficie en el proceso de oxidación, se analizaron los espectros de difracción de rayos $\mathrm{X}$ obtenidos.

La figura 4 muestra, a título de comparación, los espectros para el material base y para el material implantado con silicio. Para tiempos cortos de oxidación, se revelaron picos correspondientes a la austenita, y de posibles óxidos del tipo $(\mathrm{Cr}, \mathrm{Fe})_{2} \mathrm{O}_{3}$, en los que varían las proporciones de cromo y de hierro; identificándose también espinelas del tipo $\mathrm{Mn}_{1.5} \mathrm{Cr}_{1.5} \mathrm{O}_{4}$. A tiempos más largos de experimentación (144 h) se revela una mayor participación de las espinelas, y de más especies oxidadas del tipo $\mathrm{Cr}_{1.3} \mathrm{Fe}_{0.7} \mathrm{O}_{3}$, más que de un óxido del tipo $\mathrm{Cr}_{2} \mathrm{O}_{3}$. Las especies detectadas muestran un progresivo enriquecimiento en cromo con el tiempo de exposición, y en ningún caso se detectan picos correspondientes a óxidos de hierro pudiendo deberse a procesos difusivos que activen la formación de óxidos mixtos de cromo y de hierro.

La figura 5 muestra la sección transversal del acero AISI 304 implantado con silicio y oxidado a $1.173 \mathrm{~K}$ hasta $144 \mathrm{~h}$ de ensayo. Se observa una capa única de productos de oxidación con morfología ondulada de espesor bastante uniforme que varía entre 2 y $2,5 \mu \mathrm{m}$, observándose también la formación de una serie de nódulos distribuidos a lo largo de toda la superficie. Del espectro EDS de la capa de óxido adyacente a la superficie (Fig. 5b), se ha detectado la presencia de cromo, manganeso y hierro, aunque la intensidad de la señal de este último puede haber aumentado, debido al efecto matriz. Cabe destacar el enriquecimiento apreciable
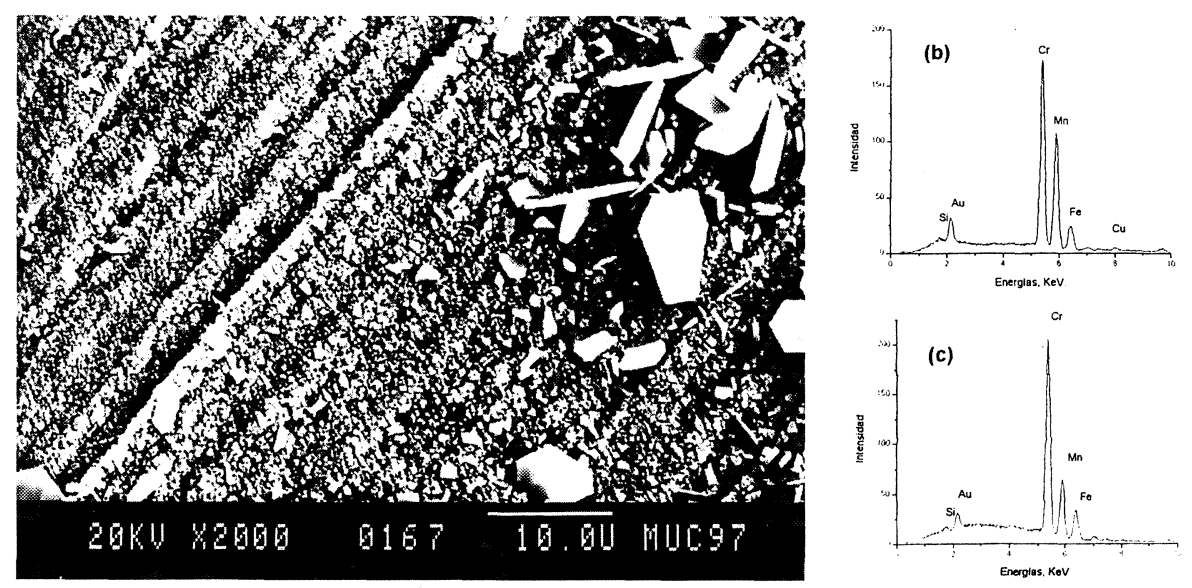

FIG. 3.- a) Morfología superficial de la capa de oxidación formada tras la oxidación a $1.173 \mathrm{~K}$ durante $144 \mathrm{~h}$ del acero AISI 304 implantado con $1 \times$ $10^{15}$ iones $/ \mathrm{cm}^{2}$ de silicio. $b$ ) Microanálisis puntual EDS de las obleas hexagonales. $c$ ) Microanálisis EDS de los cristales de pequeño tamaño.

FIG. 3.- a) Surface morphology of the oxide scale formed at 1,173 K for 144 $h$ in the $1 \times 10^{15}$ ions $/ \mathrm{cm}^{2}$ Si-implanted AISI 304 steel. b) EDS point microanalysis of the hexagonal wafers. c) EDS microanalysis of the small crystals. 


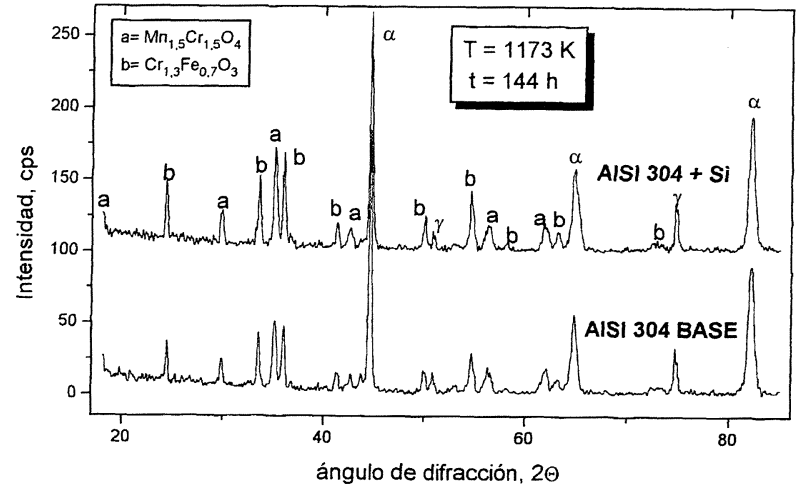

FIG. 4.- Difractogramas correspondientes al acero AISI 304 base y al implantado con $1 \times 10^{15}$ iones $/ \mathrm{cm}^{2}$ de silicio oxidados a $1.173 \mathrm{~K}$ y presión atmosférica de aire durante $144 \mathrm{~h}$.

FIG. 4.- Base and $1 \times 10^{15}$ ions $/ \mathrm{cm}^{2}$ Si-implanted AISI 304 steel diffractograms after oxidation at 1,173 K and atmospheric pressure of air for $144 \mathrm{~h}$.

en silicio, que tal y como se había propuesto anteriormente, parece incorporarse a la zona interior de la capa de oxidación. También se realizó un análisis EDS en las zonas de carburos en límite de grano, observando un espectro similar al del AISI 304 sin implantar. Pero en cuanto a la precipitación de carburos, sí parece apreciarse un efecto de impedimento en su formación en zonas adyacentes a la superficie; lo que estaría en la dirección de confirmar el posible efecto del silicio como elemento reactivo, participando en los procesos de difusión de límite de grano.

\section{CONCLUSIONES}

- La implantación con una dosis de $1 \times 10^{15}$ iones $/ \mathrm{cm}^{2}$ de silicio a $150 \mathrm{keV}$ mejora sensiblemente el comportamiento frente a la oxidación del acero AISI 304 a $1.173 \mathrm{~K}$ y presión atmosférica.

- La posible amorfización parcial en el proceso de la implantación iónica potencia el transporte de cromo en los primeros estadios del tratamiento a elevada temperatura, apareciendo numerosos núcleos en la superficie de la aleación, que posteriormente, en su oxidación, conferirán un mejor carácter protector.

- El silicio contenido en la aleación se segrega en la interfase capa-óxido e incluso se incorpora a la zona inferior de la capa o capas de oxidación formadas.

\section{REFERENCIAS}

(1) Sttot, F.H., Wood, G.C. y Stringer, J. Oxid. Met., 8, 1995: 113-145.

(2) Stringer, J. Mat. Sci. Eng., 87, 1987: 1-10.

(3) LAI, G.Y. Keynote Paper. 9 $9^{\text {th }}$ Asian-Pacific Corrosion Control Conf. Kaohsiung (Taiwan), 1995: 1-10.

(4) Huntz, A.M. Mat. Sci. Eng., 87, 1987: 251-260.

(5) Seal, S., Bose, S.K. y Roy, S.K. Oxid. Met., 41, 1994: 139-178.

(6) Saito, Y., Önay, B. y Maruyama, T. J. Phys. IV, Colloque C9, suppl. au J. de Phys. III, Vol. 3 1993: 217-230.

(7) Hussey, R.J. y Graham, M.J. Oxid. Met., 45, 1996: 349-374.

(8) Hou, P. y Stringer, J. Oxid. Met., 33, 1990: 357.

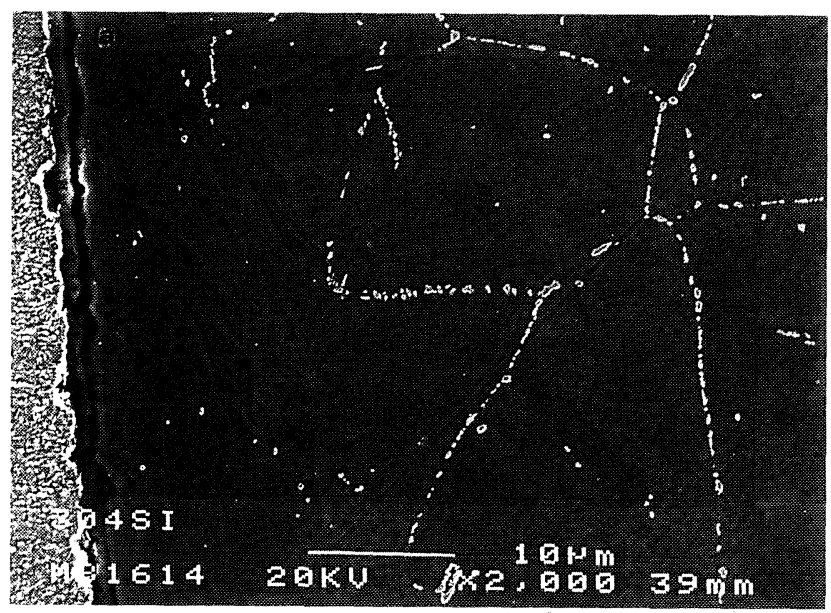

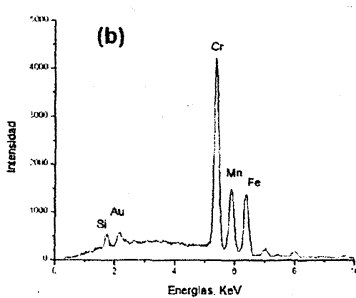

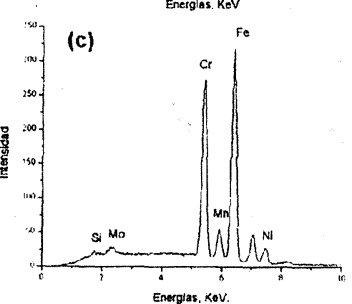

FIG. 5.- a) Sección transversal tras la oxidación del acero AISI 304 implantado con $1 \times 10^{15}$ iones $/ \mathrm{cm}^{2}$ de silicio a $1.173 \mathrm{~K}$ durante 144 h. $b$ ) Micro-análisis puntual EDS sobre los carburos precipitados en límite de grano. c) Microanálisis puntual EDS sobre carburos precipitados en límite de grano.

FIG. 5.- a) Cross-section micrograph for the $1 \times 10^{15} \mathrm{ions} / \mathrm{cm}^{2}$ Si-implanted AISI 304 steel after oxidation at 1,173 $\mathrm{K}$ for $144 \mathrm{~h}$. b) EDS point microanalysis of the oxide scale. c) EDS point microanalysis of the carbides precipitated along grain boundaries. 